\title{
The Combination of the Psycho-Pedagogical Treatment with the Neuro Biochemical Intervention in Children with Autism Spectrum Disorders
}

\author{
Ph.D. Geranda Hoxhaj \\ "Kristal" University \\ h.geranda@yahoo.com
}

\section{Doi:10.5901/ajis.2013.v2n9p526}

\section{Abstract}

Autism Spectrum Disorders have traditionally been treated as developmental disorders which affect cognitive, social and communicative ability. Scientific research is bringing in arguments that autism is caused by disorders of the immune system, diet proteins and environmental toxins. One in 88 children in the USA is affected by autism. It is now a Research Priority because of the epidemic spreading has. It is impossible to refer to statistics about the spreading of the prevalence of Autism Spectrum Disorders in Albania, as they are not complete or they do not exist. The hypothesis of this study is- the combination of the psycho-pedagogical treatment with the Neuro Biochemical intervention influences the improvement of the cognitive, social and emotional competences of children with Autism Spectrum Disorders.Methodology: This is a qualitative research based on the analysis and data collected by focus groups: parents, teachers and psychologists of children with Autism Spectrum Disorders; parents that are applying the Neuro Biochemical intervention, in confrontation with the theory of autism treatment throughout the world.The aim of this paper is to bring in a real and theoretical argument of the softening of the symptoms of autism in children with Autism Spectrum Disorders, when the Psycho-Pedagogical treatment is combined with the Neuro Biochemical intervention.

Keywords: Autism Spectrum Disorders; Psycho-pedagogical Treatment; Neuro-Biochemical Intervention; Symptoms of Autism; Softening of the Symptoms

\section{Introduction}

Autism is a bio-neurological disorder of unknown origin which affects the child's ability to process information from his or her own body or from his or her surroundings. It also interferes with a child's ability to initiate functional or communicative behavior. First described in its "pure" form by Leo Kanner $(1943,1971)$-emphasizing the impairment of the ability to make human contact or to communicate-the syndrome has been extended by the terms "pervasive developmental disorder" or "autism spectrum disorder" to cover children who show some but not all of the features initially described by Kanner. The term "spectrum" also implies that the disorder ranges from relatively mild to severe.

Autism Spectrum Disorders are complex and extremely difficult to be treated because: the cause is not completely detected yet, each individual manifests specific disorders and this is also introduced in the imbalance of biochemical tests parameters. Because of it growing dimensions, the disorder is putting before real challenges the family of the child with Autism Spectrum Disorders, the educational institutions, medical staff and the society. It is a mysterious disorder as we do not know the factors which cause it.

Autism Spectrum Disorders` treatments are divided into two major groups:

Psycho-pedagogical treatments, at the centre of which stands the treatment as "behavioral disorder or developmental disorder"

Neuro-biochemical treatments, that consider ASDs as neuro-immune disorders forced by food allergies and toxic chemicals.

These directions tend to study the same symptoms, such as: behavioral disorder, motor skills, comprehension level, eye contact, social relations and communication, stereotype and aggressiveness, etc.. Psycho-pedagogical treatment is focused on obvious external indicators, behavioral disorders, while the neuro-biochemical treatment is focused on internal causes, based on the indicators ensured by several medical tests.

There are several types of treatment known as successful around the world, according to which, children with disorders have improved their ability of adaption and self-service. It is important to highlight the fact that these have been partial changes, depending on the level of the impairment. 
Despite the well-organized work of several structures, such as, individual treatment, special education and the team work for the inclusion of children with Autism Spectrum Disorders in classrooms with normal children, the results have not been promising enough.

Project objectives. This study aims at exploring the types of the psycho-pedagogical treatment of children with ASDs. It also aims at bringing in theoretical arguments for the necessity of the neuro-biochemical intervention. This study aims at bringing in concrete arguments of the lightening of autism symptoms through the combination of psychopedagogical treatment and the neuro-biochemical intervention; in relation to the qualitative data by the focus group of parents.

Methodology. The study is a qualitative one, based on:

- Literature study focused on two courses: psycho-pedagogical treatment and neuro-biochemical intervention for children with ASDs.

- Study of the arguments on the treatments for changing or lightening autism symptoms according to respective treatments.

- Focus group of parents of children who are executing the psycho-pedagogical treatment combined with the neuro-biochemical intervention.

- Interviews with teachers of children with ASDs.

The research was focused on the information of the focus group of parents, as they were considered experts of the problematic of children with ASDs. It was chosen this methodology because of the clear validity and a clear objective and content approach and as a result, easy for everyone to understand. So, the results will be reliable. ( Marshall dhe Rossman, 2006, Merton, 1987)

The group of focus consists of 13 parents of children with ASDs. Their children are between the age of seven to ten years old. Some group members have known each other for many years and have exchanged their experiences in the treatment of these children.

As a research tool is used the combination of the semi-interview with the focus group of parents, observation and the teacher's interview.

\section{Continuing Increase in prevalence of the Autism Spectrum Disorders}

Publications of "Autism speaks.org" 2012, highlight that autism has been in the centre of media as never before. Latest updates on autism's estimated prevalence, 1 in 88 children born in America, calculated the cost of autism to society is $\$ 137$ billion per year nationally. This represents a $23 \%$ from the previous estimate, reported in 2009. It is a $78 \%$ increase from 2007. Consistent with the previous estimates, the updated numbers remains heavily skewed toward boys- 1 in 54 , compared with 1 in 252 girls. The estimated prevalence for white children was 1 in 83, compared to 1 in 227 for other communities, in 14 different communities. (www.Autism speaks.org)

March 29, 2012- the latest analysis, from a 2008 survey, shows autism is up 23\% since 2006 and 78\% since 2002. With nearly a doubling of prevalence since CDC started tracking in 1992, autism is officially becoming an epidemic in the U.S.. More autism research and better services for people living with autism will be expensive. But the cost of autism already is astronomical. At the new 2008 prevalence rate of one in 88 American children, autism costs the U.S. \$137 billion a year. It has been estimated that $45 \%$ of Americans with autism have an intellectual disability. The lifetime cost for each person who has an intellectual disability related to autism is $\$ 2.3$ million. (https://stopcallingitautism.org /prevalenceandcost.html)

16 million children are diagnosed with concentration, behavioral and learning problems. The number of those diagnosed with ADHD, Asperger's syndrome, Dyslexia, Tourette`s syndrome, Bipolar disorder and any other obsessive compulsive disorder. It is a new phenomenon and the most important issue of these days. Only ten years ago, autism was considered a rare disorder, diagnosed in 1 in 10.000 children born in the USA. ( Disconnected Kids.Dr.Robert Melillo.2010.pg.6)

Autism has affected 1 in 110 in 2009, 1 in 88 in 2011 and 1 in 50 are now affected, in 2013!

The annual cost in the United States is 90 billion dollars ( $90 \%$ of the cost for adult care and services). In 10 years (2017), the annual cost of autism will be $\$ 200-400$ billion. (https://stopcallingitautism.org/content/presentations/JuanRodriguez-SCIA-AutismOne-2013.pdf)

In Albania, we lack statistics, though, the problem is evident. The yeras of sacrifies made by parents on behalf of their children with autism- without proffesional training- make the big difference between children with autism in Albania and those in developed country; moreover, without a specific diagnose of the Autism Spectrum Disorders. 


\section{The psycho-pedagogical treatment of children with Autism Spectrum Disorders between clash of ideas and arguments.}

The psycho-pedagogical treatments of these days are facing each other; each representative has conceived and achieved a certain treatment from his point of view.

- The Applied Behavior Analysis (ABA), is defined as the science in which the principles of the behavior analyses have been systematically applied to improve socially important behaviors- where experiments are used to identify the responsible variables for the behavioral changes. (Cooper et al.. Applied Behavior Analysis. p. 20. ISBN 0-675-20223-X) "ABA is based on the traditional theory of behaviorism, to change men's behavior as part of the learning or treatment process.". ("Positive Behavior Support and Applied Behavior Analysis". The Behavior Analyst 29 . Retrieved January 12, 2013

Even though ABA is considered as successful, in 2011, investigators from the Vanderbilt University, in cooperation with the Agency for Healthcare Research and Quality (AHRG), performed an overall survey of the fundamental scientific literature during APA and other therapies for Autism Spectrum Disorders, including UCLA/Lovaas Method and the Early Start Denver Model. They concluded that "both approaches have improved referring to the comprehension performance, communicative skills and other adaptive behavior ability." They also assumed that "the power of evidence was low, many children continue to display damaged areas, subgroups may compose the majority of the change; it is not the evidence for the practical efficiency beyond the research studies and the published studies, have used small samples, various approaches and treatment duration, and above all, different final measurement." (Warren, Z.; Veenstra-VanderWeele, J.; Stone, W.; Bruzek, J.L.; Nahmias, A.S.; Foss-Feig, J.H.; Jerome, R.N.; Krishnaswami, S.; Sathe, N.A.; Glasser, A.M.; Surawicz, T.; McPheeters, M.L. (2011). Therapies for children with autism spectrum disorders. AHRQ Publication No. 11-EHC029-EF, Comparative Effectiveness Review No. 26. Rockville, MD: Agency for Healthcare Research and Quality).

- The Miller Method is based on the theory of systems and it stresses the need to be based on them and to enrich the capacity of the child. The single most unique and important aspect of the Miller Method is its work with systems. The Miller Method contrasts sharply with applied behavior analysis (ABA).Where ABA requires the child to remain seated to learn, the MM assumes that children with autism learn best through action; where ABA emphasizes "turning away" from tantruming or acting-out children, the MM emphasizes turning toward and engaging the child; where ABA tries to divert or "extinguish" aberrant behavior, the MM attempts to transform these behaviors into functional, interactive exchanges. While the ABA approach often achieves the child's compliance with its methods, its emphasis on compliance seems at odds with the child's need to cope in an inconstant world and-equally important-with the kinds of bioneurologically based challenges these children experience. (The Miller Method, Arnold Miller with Kristina Chrétien2007 pg.17)

- Brain Balancing program. Dr. Robert Melillo argues that, "based upon my studies, I have had the possibility to notice that disorders affect every system of the body, not only the brain. The majority of professionals are still treating it as a single problem, such as concentration, learning, socialization, or other symptoms. Although, the majority of these kids manifest a combination of several symptoms including different systems: sensory, motor, cognitive, emotional and immune system." ( Disconnected Kids. Dr. Robert Melillo. 2010. pg.15)

"Every disorder is a complex one, and often it includes every body system. All the circumstances which negatively affect behaviors and learning, are subject to the imbalance electrical problems. The result is that a child with brain imbalance, possesses normal or even extremely normal skills regarding the higher brain function, and extremely bad ability, regarding the lower brain." (Disconnected Kids Dr. Robert Melillo 2010 .pg. 29)

"the Brain Balancing Program is based upon a technique named the Hemispheric Integration Therapy. There have been selected several daily exercises- sensory, physical and academic- which operate directly upon the problems." ( Disconnected Kids Dr. Robert Melillo 2010. pg 118)

- Individual therapy- applied in our country, treats the child as a person who has to learn some activities or behaviors- by repetition of the same activities, or by miming the therapist. It is supposed that the major problem of individual therapy, is that the improvement does not go further than therapies' environment. Here in Albania the so-called "developmental therapy" is performed by one therapist; there does not exist a team of therapists, or even an individualized plan; she is not supervised by specialized institutions or state structures. 
Within this paper are included those theories that are considered interesting according to the way they are conceptualized and performed. Although we are offered principles and theories, we lack statistics of treated children and we consequently lack the level of the treatment's efficiency. But one thing seems to be evident, the results cover just one part of the case; on the other hand, the number of those waiting for a more efficient treatment is ever-growing.

\section{The neuro-biochemical intervention based upon scientific research- a new possibility for the treatment of children with Autism Spectrum Disorders.}

The unsatisfactory results of the psycho-pedagogical treatment, lead to another possible treatment. The almost epidemic prevalence of autism spectrum disorders has placed it as a priority for the scientific research, focused on the acquired autoimmune systems, low detoxification capacity for definitely environmental causes, inflammatory bowel disease or other microbiological co-factors.

In the recent years, autism has been an issue of interest for talented scientists from other scientific fields. In 2012, many of these teams published important discoveries, confirmed and based upon previous pioneering discoveries. High priority is given to the field of science and autism this year. Research teams in every country of the world are involved within this improvement. Some improvements are focused in new areas of research. (www.Autism speaks.org)

- Autism Research Insitute, established in 1967 by the research psychologist, Bernard Rimland, Ph.D.,in the USA. ARI is a major proponent of the biomedical approach for autism treatment. The premise for integrative medical intervention is that certain neurological disorders, including autism, might be caused by environmental triggers that compromise the gastrointestinal, immunological, and neurological systems. (Biomedical Treatments for Autism from the Autism Research Institute 2008). The DAN (Defeat Autism Now) treatment shows the relation between autism symptoms and their causes. Normal organism can eliminate the heavy metals from the system- with the help of Glutathione enzymes which consists of Casein. In children with autism these elements are found in a low level. Consequently, children who are likely to have autism possess high toxic levels. Heavy metals prefer fatty setting, fat makes up $60 \%$ of our organism. This percentage explains the existence of the relation between heavy metals and brain. The presence of mercury causes difficulty in social relationship, lack of eye contact, stereotypical behaviors, speaking retardation, etc. Various heavy metals have been considered as the origin of autism, such as: mercury, leaden, aluminum, nickel and arsenic. Costa LG, Aschner M, Vitalone A, Syversen T, Soldin OP. Developmental neuropathology of environmental agents. Annu Rev Pharmacol Toxicol 2004; pg.87).

The detoxification suggested by DAN is achieved through the neuro-biochemical supplement, therapies of oxygen and food diet.

- Stop Calling it Autism. It considers autism as a disorder, characterized by neuro-developmental impairments, important for the behavior, communication and social function. Evidence shows that children with autism suffer from a continuous neuro-inflammatory process, in different areas of the brain, which include the Microglial Activation. The Microglial Activation is accompanied by loss of connection between neurons by reducing their function and leading to the well-known "autism". SCIA is based on "the connection between mental diseases and the immune impairment", by Mario Cappecchi, the winner of the Nobel Price 2010. Future studies must inspect treatments that can reduce the microglial activation and neuro- inflammation or that can finally lighten ASDs symptoms." (Cambridge Journals, May 2011, "Evidence of microglial activation in autism and its possible role in brain under connectivity" Juan I. Rodriguez and Janet K. Kern). The impaired immune system prior to the SCIA, is considered as a concern to all researches of various scientists on this field. The way the treatment is performed, makes the difference.

- Additional arguments about the relation between disorders and eating. Historically, many doctors have not agreed that diets, food ingredients, sugar, etc. can influence behaviors. They admitted that it did not exist real scientific evidence for that. But in 2008 this viewpoint altered. It was agreed and published by the American Academy of Pediatrics in the "ADHD and Food Additives Revisited", by Alison Schonwald. Thousands of parents decided to implement diets and they have observed considerable improvement. (Knivsberg, REICHELT, et al. A randomized, controlled study of dietary intervention in autistic syndromes Neuroscience 5 nr.4 (2002) 251-256)

Brain Development and function. Your child's brain is highly dependant on the vitamins, minerals, amino acids, essential fatty acids, and calories found in food. For instance, if your child is not getting enough of certain key nutrients, it compromises his neurotransmitter production, the synthesis of his brain`s main myelin 
sheath, glucose oxidation, and his visual and cognitive processing. If he`s consuming too much sugar and artificial additives, it can compromise his brain function and contribute to behavior and learning problems.

In the 1980's, researchers found evidence of food proteins in the urine of autistic children that resemble opioids. Opioids are substances that can cause behavioral changes in people. (An example is the drug morphine, which is derived from opium). Opioids proteins are known to attach to receptors in the brains and guts to create behavioral changes as well as digestive complaints like constipation, diarrhea and bloating.

"Leaky gut" is common in autism and implies that the intestines are more permeable than normal. This can play a major role in food allergies allergy and in soy, gluten and casein soy, and gluten and casein sensitivity.

Soy, gluten and casein can enter the circulation through this "leaky gut" and travel to the brain. Gastrointestinal Symptoms in Autism are: Abdominal Pain, Chronic Diarrhea, Constipation, Gaseousness/Bloating, Nighttime Awakenings, and Unexplained Irritability. (http://www.cemkinaci.com)

Additionally, our brain balancing program includes nutritional guidelines - as diet problems are one of the causes of the evolution of neurological disorders during childhood. It manifests the environmental factors related to the problem. This program enables the surveillance of parents, teachers and staff's activity to achieve stable results. Physical, mental and eating activities, will lead to gradual change in the academic work and the child's brain changes and starts normal development.

The expected results are: Academic performance improvement, even the highest marks, reduction of negative behavior, elimination of irritating or extreme behavioral, communicational progress and social ability. (Disconnected Kids. Dr. Robert Melillo. 2010. Fq. 110 - 123)

- GcMAF - the beginning of the end for autism Over 900 children have now trialed GcMAF around the world, and clinics are reporting a response rate of over $80 \%$ in reducing the symptoms of autism. (PRWEB UK September, 8 2012). GcMAF is a key component of our immune system: all healthy people have it, people with chronic diseases do not. "Immune Biotech Ltd" extracts and purifies GcMAF in their Oxford laboratories.

- Data from our focus group of parents of children with Autism Spectrum Disorders illuminate the combination of the psycho-pedagogical treatment and the neuro-biochemical intervention.

Our focus group has answered the semi-structured questions through discussion and exchange of experiences.

How long has the child attended the developmental therapy? What changes were noticed? It was stated by the focus group that children had started the developmental therapy after the second or third year of their life. Disconnections from these therapies were frequent in cases when parents did not notice any changes. They then were in search of other therapists. Two boys had attended one therapist for many years, as the parent had noticed progress and that the children could learn, although very slowly; he only paid attention to what she said. One of the girls with the disorder had had the chance to become part of an ABA program, from respective specialists, in America, who had programmed to monitor her performance. Due to this therapy, she has made progress in her vocabulary and in her ability to link words in a sentence.

Have you ever tried a diet? What have you noticed? All parents had tried to implement diets according to information from different origin, or by medical prescriptions. It had not been possible for some parents to implement the diet, as a result of their way of living, or because of the inability to adopt the child with the new food regime. Other parents had achieved to implement just half of the diet, by eliminating just the white sugar or casein. The other group of parents had been implementing the whole diet for two years now; sugar free, casein free and gluten free. The diet had lead to several changes, for instance, partial decreased hyperactivity, sleep stabilization or a more quiet condition.

Why and how have you performed the neuro-biochemical intervention? Parents admitted that as they needed treatment and years were flying away, they had the necessity to have information from every part of the world. Due to technological improvements, they are informed in real time about every scientific research on autism. Parents have contacted doctors from abroad, such as, in Italy, England, Turkey, and Greece.

Some parents have the same doctor as mentor for- the neuro-biochemical treatment, or the supplement, culinary diet or hyperbaric therapy. (HOBOT) It varies from one to two years, in different children. While parents are still under this process, they range these changes: reduced hyperactivity, improved your concentration, higher level of comprehension, higher level of cooperation, higher level of stability reducing stereotype threat. In a few words, these are positive changes, but it is not clear yet to what extent will this condition improve? 
It was stated by the teachers ' interview, that they had noticed improvement in the level of comprehension, and a reduction in stereotype threat and irritation in children under the effect of the neuro-biochemical intervention. Teachers noticed a big difference between children that had gone in a diet, that were under the neurobiochemical treatment and those who did not undergo this treatment.

\section{Conclusion}

All the data gathered by the focus group of parents of children with Autism Spectrum Disorders, serves as an evidence for the fact that the combination of the psycho-pedagogical treatment and the neuro-biochemical intervention, influence the improvement of the cognitive, social and emotional skills of children with Autism Spectrum Disorders.

\section{References}

Marshall and Rossman, 2006, Merton, 1987

https://stopcallingitautism.org/content/presentations/Juan-Rodriguez-SCIA-AutismOne-2013.pdf

Cooper et al. Applied Behavior Analysis, pg. 20 ISBN 0-675-20223-X).

"Positive Behavior Support and Applied Behavior Analysis". The Behavior Analyst 29 . Retrieved January 12, 2013

Warren, Z.; Veenstra-VanderWeele, J.; Stone, W.; Bruzek, J.L.; Nahmias, A.S.; Foss-Feig, J.H.; Jerome, R.N.; Krishnaswami, S.; Sathe, N.A.; Glasser, A.M.; Surawicz, T.; McPheeters, M.L. (2011).

Therapies for children with autism spectrum disorders. AHRQ Publication No. 11-EHC029-EF, Comparative Effectiveness Review No. 26. Rockville, MD: Agency for Healthcare Research and Quality

The Miller Method, Arnold Miller with Kristina Chrétien.2007

www. autismspeaks.org

Biomedical Treatments for Autism from the Autism Research Institute 2008

Costa LG, Aschner M, Vitalone A, Syversen T, Soldin OP. Developmental neuropathology of environmental agents. Annu Rev Pharmacol Toxicol 2004; fq.87

Cambridge Journals, maj 2011, "Evidence of microglial activation in autism and its possible role in brain underconnectivity" Juan I. Rodriguez and Janet K. Kern

Eating for autism. Elizabeth Strickland 2009. pg. 1

http://www.cemkinaci.com

Disconnected Kids. Dr. Robert Melillo. 2010

PRWEB UK September 8, 2012 